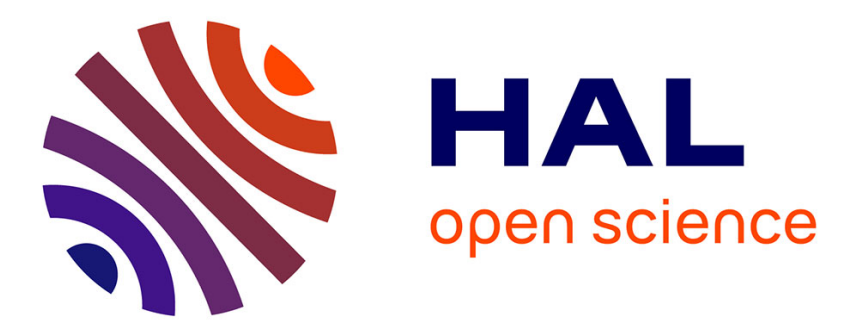

\title{
Utilisation d'un attractif de synthèse pour la surveillance et le piégeage des pyrales Phycitinae dans les locaux de stockage et de conditionnement de denrées alimentaires végétales
}

\author{
Francis Fleurat-Lessard, Marie-Pierre Siegfried, Jean-Marc Le Torc'H
}

\section{To cite this version:}

Francis Fleurat-Lessard, Marie-Pierre Siegfried, Jean-Marc Le Torc'H. Utilisation d'un attractif de synthèse pour la surveillance et le piégeage des pyrales Phycitinae dans les locaux de stockage et de conditionnement de denrées alimentaires végétales. Agronomie, 1986, 6 (6), pp.567-578. hal-00884911

\section{HAL Id: hal-00884911 \\ https://hal.science/hal-00884911}

Submitted on 1 Jan 1986

HAL is a multi-disciplinary open access archive for the deposit and dissemination of scientific research documents, whether they are published or not. The documents may come from teaching and research institutions in France or abroad, or from public or private research centers.
L'archive ouverte pluridisciplinaire $\mathbf{H A L}$, est destinée au dépôt et à la diffusion de documents scientifiques de niveau recherche, publiés ou non, émanant des établissements d'enseignement et de recherche français ou étrangers, des laboratoires publics ou privés. 


\title{
Utilisation d'un attractif de synthèse pour la surveillance et le piégeage des pyrales Phyci- tinae dans les locaux de stockage et de condi- tionnement de denrées alimentaires végétales
}

\author{
Francis FLEURAT-LESSARD \\ avec la collaboration technique de Marie-Pierre SiegrRied $\left({ }^{*}\right)$ \& Jean-Marc LE TORC'H
}

I.N.R.A., Laboratoire des Insectes des Denrées, Station de Zoologie, Centre de Recherches de Bordeaux, F 33140 Pont-de-la-Maye

(*) Institut de Recherches Tézier, F 26003 Valence

\begin{abstract}
La protection des stocks contre les attaques de lépidoptères Pyralidae, de la sous-famille des Phycitinae, au moyen d'un attractif de synthèse actif sur les principales espèces nuisibles est abordée par plusieurs procédés. Le piégeage d'avertissement au moyen des pièges disposés aux endroits sensibles des entrepôts renseigne efficacement sur les risques d'une première infestation. Cette technique est généralement accompagnée de traitements insecticides des locaux contaminés. La confusion sexuelle des mâles provoquée par la diffusion de fortes doses de phéromone sexuelle a des effets incomplets sur la rupture des communications sexuelles. Elle est relativement coûteuse à entretenir en permanence dans les entrepôts. Le piégeage en masse, réalisé au moyen d'un réseau dense de pièges à phéromone, permet de localiser l'infestation et d'éliminer progressivement les insectes qui émergent des denrées contaminées.

Les possibilités d'utilisation pratique en entrepôt de fruits secs, dans les moulins, etc..., du dernier procédé en particulier, avec des pièges spécifiques, sont discutées.
\end{abstract}

Mots clés additionnels : Piégeage en masse, confusion sexuelle, Plodia interpunctella, Anagasta kuehniella, Ephestia elutella, Cadra cautella. situations.

Storage systems provide a favourable situation for pheromone use. Three ways of controlling Phycitinae moth populations were investigated in different storage buildings. The attractive sex pheromone of Phycitinae was used either for moth population surveys, or for mating disruption or mass trapping. Trials with the single synthetic pheromone which is attractive to all males of Phycitinae which are storage pests showed that moth infestations could be greatly reduced by 2 operating processes (but not with the confusion technique) : coupling of low trapping pressure with localized insecticidal disinfestation of the warehouse and mass trapping of males when insecticide use is not recommended.

The prospects for use of specific pheromone traps in stores, without insecticide, are presented.

Additional key words : Mass-trapping, mating disruption, Indian meal moth, Mediterranean flour moth, tobacco moth, almond moth.

\section{INTRODUCTION}

Pour les insectes, les phéromones sont à l'origine d'une des formes essentielles de communication et souvent la plus efficace à distance. La plupart des phéromones sexuelles connues de Lépidoptères nuisibles aux denrées entreposées sont produites par les femelles, ce qui permet leur localisation précise par les papillons mâles de la même espèce. C'est le cas pour les pyrales de la sous-famille des Phycitinae, dont plusieurs espèces sont, au stade chenille, des ravageurs importants des fruits secs, des semences, de la farine et d'autres produits végétaux secs pendant la période de conservation. En France, ces infestations concer- 
nent, pour l'essentiel, des denrées alimentaires trans formées sur lesquelles aucune application directe d'insecticide, ni gazage ne sont tolérés. La pratique de la désinsectisation préventive des locaux d'entreposage vides (inoccupés) ne peut suffire à réduire suffisamment les infestations pendant toute la durée de la conservation. Les chenilles de papillons Phycitinae constituent donc un risque potentiel de dégradation de l'état sanitaire dans les principaux cas suivants :

- dans les stocks de pruneaux et autres fruits secs (noisettes, amandes, noix, figues, etc...) produits en France ou importés et stockés de façon traditionnelle ; les attaques de la "teigne des fruits secs», Plodia interpunctella (Hübn.) et, dans une moindre mesure, celles de la "teigne des amandes », Cadra cautella (Walk.) sont à redouter de mai à octobre ;

- dans la minoterie, semoulerie et boulangerie industrielle, la "mite de la farine », Anagasta (= Ephestia) kuehniella (Zell.) a été longtemps considérée comme la «peste des moulins ». Bien que les cas de présence dans les moulins soient en nette régression, le risque demeure, en particulier en boulangerie et pâtisserie et les conséquences d'une multiplication de ce ravageur peuvent être graves pour l'entreprise ;

- dans les ateliers de conditionnement de semences ou de végétaux séchés (herboristerie, plantes officinales ou aromatiques, etc...), se produisent des infestations sporadiques de $P$. interpunctella, $A$. kuehniella et $C$. cautella ; chaque insecte vivant dans la marchandise représente une imperfection inacceptable pour ces produits à forte valeur ajoutée ;

- sur des produits exotiques comme le cacao, on peut rencontrer la «pyrale du cacao », Ephestia elutella (Hübn.), bien qu'elle soit actuellement supplantée par la "mite du riz», Corcyra cephalonica (Staint.) qui fait partie de la sous-famille des Galleriinae. Néanmoins, E. elutella demeure un ravageur potentiel des feuilles de tabac séchées.

Grâce aux travaux de nombreuses équipes de recherche en chimie et en entomologie, on a montré, au cours des 15 années écoulées, que les substances phéromonales émises par les femelles des 4 espèces citées avaient un composé attractif principal qui leur était commun, l'acétoxy-1 tétradécadiène 9-Z, 12-E appelé successivement "Prodenialure B », «ZETA » ou «TDA». Le terme commun de prodenialure est issu du nom de genre d'une noctuelle, Prodenia eridania (Cram.) dont la phéromone comporte 2 substances principales en mélange. L'une d'elles, le «ZETA », s'est avérée par la suite être la substance attractive principale des Phycitinae. L'abréviation chimique la plus en usage actuellement est Z9, E12-14 : Ac. Les autres substances présentes dans le « bouquet phéromonal » de ces espèces sont, pour l'essentiel, des isomères géométriques du ZETA ou des dérivés inhibiteurs ou synergistes du composé principal évitant les méprises entre individus d'espèces différentes (tabl. 1).

TABI EAU I

Phéromones des principales especes de pyrales Phycitinae.

Pheromones of the main species of Phycitinae moths.

\begin{tabular}{|c|c|c|c|}
\hline Espèces & Effet & Phéromones & Auteurs \\
\hline $\begin{array}{l}\text { Plodia interpunctella } \\
\text { (teigne des fruits secs) }\end{array}$ & $\begin{array}{l}\text { attractif } \\
\text { synergiste }\end{array}$ & $\begin{array}{l}\text { (1) Acétoxy-1 tétradécadiène 9-Z, 12-E } \\
\text { (2) Tétradécadiène 9-Z, 12-E, ol-1 }\end{array}$ & $\begin{array}{l}\text { KuWAHARA et al. } \\
(1971 a) \text {, } \\
\text { BRADY et al. } \\
(1971)\end{array}$ \\
\hline & $\begin{array}{l}\text { inhibiteur } \\
\text { inhibiteur }\end{array}$ & $\begin{array}{l}\text { (3) Acétoxy-1 tétradécène 9-Z } \\
\text { (4) Acétoxy-1 létradcicadiènc 9-Z, 12-E }\end{array}$ & $\begin{array}{l}\text { BRADY (1969) } \\
\text { VICK \& SOWER } \\
(1973)\end{array}$ \\
\hline $\begin{array}{l}\text { Ephestia (= Cadra) cautella } \\
\text { (teigne des amandes) }\end{array}$ & $\begin{array}{l}\text { attractif } \\
\text { synergiste } \\
\text { inhibiteur }\end{array}$ & $\begin{array}{l}\text { (1) Acétoxy-1 tétradécadiène } 9-Z, 12-\mathrm{E} \\
\text { (3) Acétoxy-1 tétradécène } 9-Z \\
\text { (2) tétradécadiène } 9-Z, 12-E, \text { ol-1 }\end{array}$ & $\begin{array}{l}\text { READ \& BEEVOR } \\
(1976)\end{array}$ \\
\hline $\begin{array}{l}\text { Ephestiu elutella } \\
\text { (reigne du caciulo) }\end{array}$ & attractif & (1) Acétoxy-1 tétradécadiène 9-Z, 12-E & \\
\hline $\begin{array}{l}\text { Ephestia (= Anagasta) kuehniella } \\
\text { (mite de la farine) }\end{array}$ & $\begin{array}{l}\text { attractif } \\
\text { inhibiteur }\end{array}$ & $\begin{array}{l}\text { (1) Acétoxy-1 tétradécadiène } 9-Z, 12-E \\
\text { (3) Acétoxy-1 tétradécène } 9-Z\end{array}$ & $\begin{array}{l}\text { KUWAHARA et al. } \\
(1971 b)\end{array}$ \\
\hline $\begin{array}{l}\text { Ephestia (= Cadra) figulilella } \\
\text { (teigne des raisins secs) }\end{array}$ & altractif & (1) Acétoxy-1 tétradécadiène 9-2, 12-E & $\begin{array}{l}\text { BRaDy \& Daley } \\
(1972)\end{array}$ \\
\hline
\end{tabular}




\section{MATÉRIEL ET MÉTHODES}

L'utilisation pratique de la phéromone sexuelle de synthèse des papillons Phycitinae dans les entrepôts et les magasins de conditionnement des denrées s'est heurtée à 3 problèmes majeurs, qui ont été largement étudiés au cours des 10 dernières années :

- il n'est pas possible de montrer une relation directe entre la population attirée vers les pièges appâtés avec le produit attractif sexuel et le niveau réel de la population totale présente dans le stock à ce moment-là. Chaque espèce réagit selon son comportement et celui-ci évolue avec l'âge, les facteurs du milieu environnant et les conditions du piégeage ;

- en corollaire, la technique d'échantillonnage de la population de papillons mâles, par l'intermédiaire d'un attractif modifiant leur comportement habituel ne donne pas des résultats significatifs directement utilisables pour la détermination des risques encourus. L'interprétation du nombre de captures est délicate pour décider de l'opportunité d'interventions insecticides ;

- l'utilisation du piégeage en tant que méthode de lutte nécessite des connaissances approfondies sur le comportement des différentes espèces dans des situations extrêmement variées comme celles que l'on peut rencontrer dans les manufactures et entrepôts de denrées alimentaires.

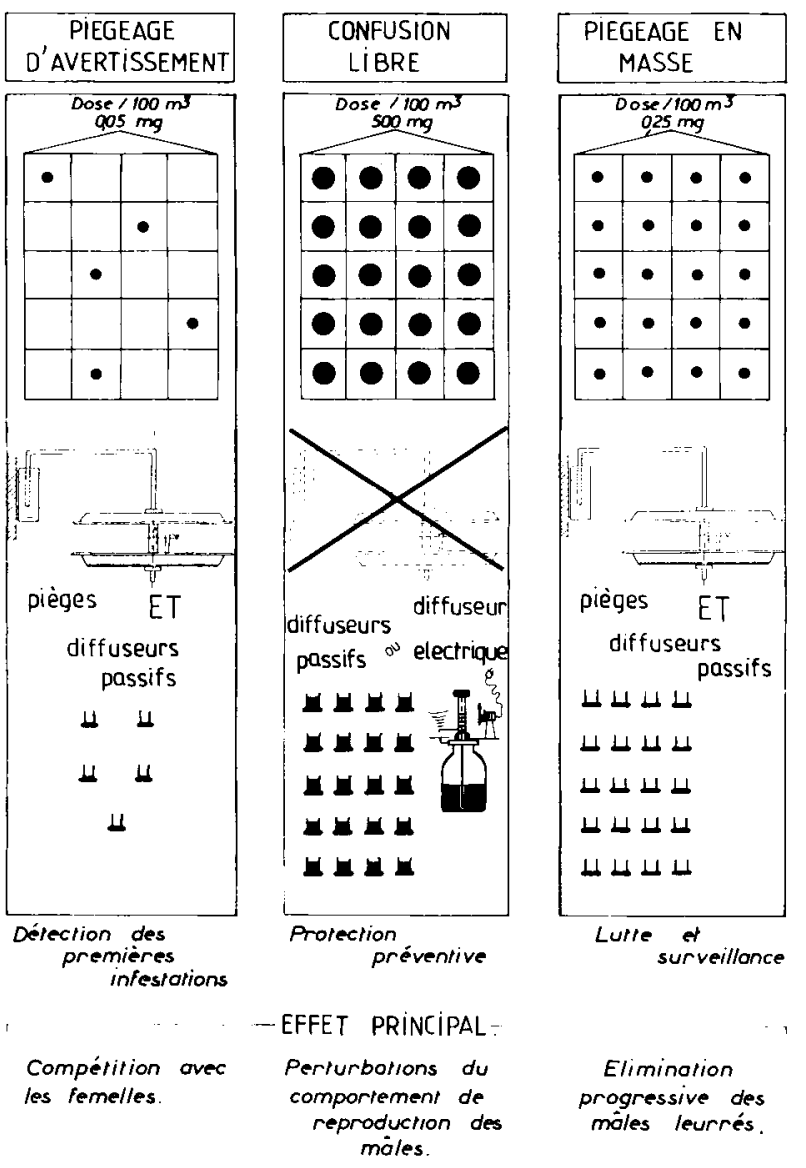

Figure 1

Description schématique des différentes possibilités d'utilisation des phéromones sexuelles de lépidoptères en entrepôts de denrées.

Schematic picture of the different means for use of sexual pheromones for the control of stored products moths in warehouses.
En accord avec les conceptions théoriques énoncées dès 1976 par HAINES, on peut affirmer qu'il existe 3 façons différentes d'utiliser des phéromones sexuelles de lépidoptères en entrepôt de denrées alimentaires avec ou sans dispositif de piégeage (fig. 1) :

1) La surveillance ( monitoring ») des infestations, employée à titre préventif avec des pièges dispersés aux points sensibles des entrepôts (stock ancien, réserve d'emballages en carton ondulé, denrées exposées à la chaleur, produits très aromatiques, etc...) et contenant des diffuseurs à faible émission $(0,1$ à $0,2 \mathrm{ng} / \mathrm{j})$.

2) Le piégeage en masse de papillons mâles ( $«$ masstrapping $")$ avec un réseau dense de pièges et avec des diffuseurs du premier type devrait permettre la diminution progressive de la population par élimination régulière des mâles qui émergent des denrées ou du local.

3) La diffusion active ou passive de quantités importantes de phéromone de synthèse $(2 \mathrm{~g} / \mathrm{j}$ pour $1000 \mathrm{~m}^{3}$ ) dans l'atmosphère de l'entrepôt qui permet, dans le meilleur des cas, d'empêcher la rencontre des sexes.

Depuis 1975, nous avons expérimenté ces 3 techniques en laboratoire puis dans les bâtiments de stockage et de conditionnement, principalement pour contrôler les populations de $P$. interpunctella dans les entrepôts traditionnels de fruits secs (PIMAUD et al., 1976 ; FLEURAT-LESSARD et al., 1976).

\section{RÉSULTATS ET DISCUSSION}

\section{A. Le piégeage d'avertissement}

Les premières expérimentations ont concerné essentiellement le procédé de piégeage et la mesure de la diffusion des produits de synthèse placés dans les diffuseurs. On a pu observer successivement que la couleur des pièges n'a pas influencé la capture des teignes des fruits secs et que, quelle que soit la quantité de produit déposée dans le diffuseur, des papillons mâles sont capturés alors que les pièges témoins, sans attractif, ne capturent rien ou seulement de rares individus pris au hasard. La forme des pièges a évolué régulièrement pour combiner une bonne efficacité avec un montage simple et un coût le plus bas possible. On a abouti actuellement à un montage constitué de 2 assiettes en plastique blanc, disposées face-à-face, dont l'écartement est maintenu par un tube plastique rigide enfilé sur la tige servant de support et d'axe central pour le piège (fig. 2). Seule l'assiette inférieure est enduite de glu. Ce modèle, qui peut être monté entièrement sur place, nous a paru préférable à d'autres dont la facilité d'emploi est plus contestable (LEVINSON \& LEVINSON, 1979).

Des combinaisons de produits attractifs différents ont été tentées avec un $2^{\mathrm{e}}$ composé émis par les femelles de $P$. interpunctella, le tétradécadiène $9-Z, 12-\mathrm{E}$, ol-1 appelé communément TD ol ou Z9, E12-14: $\mathrm{OH}$, abréviation la plus usitée actuellement. Cette substance est inhibitrice de l'attraction des mâles de 


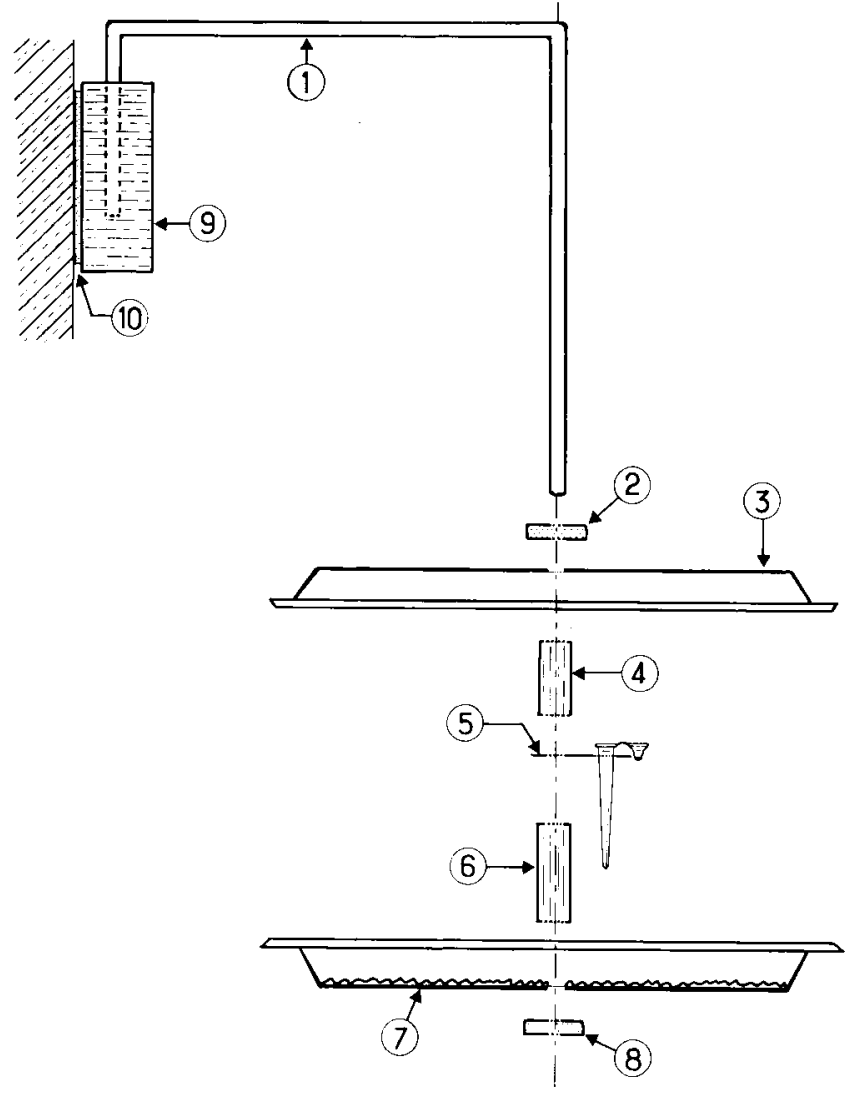

Figure 2

Schéma d'assemblage du piège expérimental I.N.R.A. pour la capture des papillons mâles de Phycitinae dans différentes situations d'entreposage de denrées alimentaires.

Construction of the I.N.R.A. model of pheromone trap to catch the Phycitinae moths in the different situations of food storage.

Légende (dans l'ordre de montage) : 1 : tige portoir ; 2 : rondelle de caoutchouc (butée) ; 3 : assiette lisse retournée; $4:$ tube court en plastique transparent ; 5 : lamelle à 2 trous porte-diffuseur ; $6:$ tube long; 7: assiette enduite de glu; 8: rondelle de caoutchouc (serrage); 9 : carrelet de bois à trou central ; 10: bande adhésive de fixation aux supports lisses verticaux.

C. cautella ce qui justifiait la $1^{\text {re }}$ appellation de $«$ cautelhib ». Nous avons déjà démontré indirectement que la volatilité différente des 2 composés limitait les avantages de leur association sur le plan pratique (LE TORC'H \& FlEURAT-LESSARD, 1980). La synergie qui existe pour la capture de $P$. interpunctella, lorsque les 2 composés attractifs sont en proportion définie, ne se manifeste que pendant une courte durée après l'installation des diffuseurs. Cette association a été expérimentée depuis pour rendre le piégeage absolument sélectif pour une seule espèce nuisible (VICK et al., 1981). Avec la diversité des sites d'utilisation des pièges à phéromone pour la surveillance des infestations de papillons Phycitinae, il est apparu avantageux de pouvoir capturer plusieurs espèces avec la même substance et le même type de piège.

Les doses de produit à placer dans les diffuseurs ont été déterminées par l'expérience en tenant compte de certaines particularités du comportement et de la biologie des espèces à capturer : les papillons sont actifs seulement aux périodes sombres de la journée (en général le matin pour $E$. kuehniella et le soir pour $P$. interpunctella) et la diffusion de phéromone pen- dant la photophase, période habituelle de repos des papillons, perturbe leur comportement normal. Des doses de $10 \mu \mathrm{g}$ par diffuseur ont été adoptées pour la mite de la farine et une charge initiale de $5 \mu \mathrm{g}$ convient pour les autres espèces. Néanmoins, plus la dose est élevée, plus le nombre de captures est important (l'effet principal paraît lié à l'augmentation du rayon d'action des pièges). Le solvant choisi a été successivement le dichlorométhane, le trichloro $1,1,1$, éthane ; c'est actuellement l'hexane pur. L'association de la phéromone, qui est un liquide à consistance huileuse, avec un solvant volatil permet l'imprégnation des diffuseurs en caoutchouc ou en polyéthylène. Ceux-ci émettent ensuite passivement le produit dans l'air, de façon plus ou moins régulière, selon les conditions physiques de l'environnement.

L'emplacement des pièges est primordial pour un bon rendement. Les stocks alimentaires fixent les papillons par leurs arômes et, dans le cas des espèces les moins mobiles, l'espace de déplacement des papillons autour des stocks est restreint à quelques mètres (FLEURAT-LESSARD, 1983). De même, le rayon d'action des pièges est réduit à $1 \mathrm{~m}$ en moyenne, en espace absolument calme (MANKIN, 1979). La diffusion mesurée par la perte de poids d'une capsule de diffusion chargée de $10 \mu \mathrm{g}$ de phéromone peut atteindre $20 \mathrm{ng} / \mathrm{j}$ au début et seulement $2 \mathrm{ng} / \mathrm{j} 3$ mois après (mais elle libère encore, un an après, quelques traces de produit qui sont cependant suffisantes pour assurer quelques captures).

L'interaction relevée entre l'emplacement du piège et la quantité de substance placée dans chaque diffuseur est significative d'un manque d'efficacité du piège en position éloignée du stock (fig. 3) pour la teigne des fruits secs dans un stock de pruneaux.

L'accroissement régulier des captures, entre 2 relevés successifs des pièges, est l'indice d'une infestation

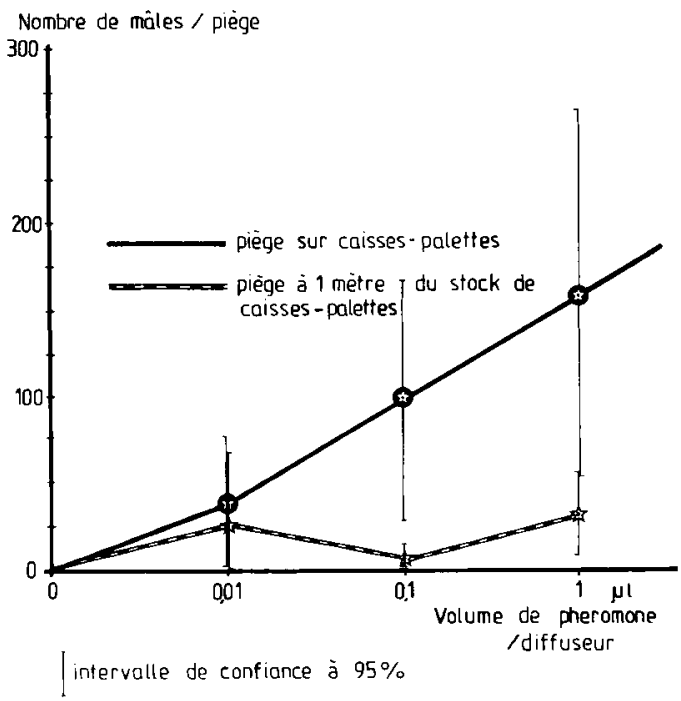

Figure 3

Efficacité des pièges à phéromone sexuelle de synthèse de $\mathrm{P}$. interpunctella dans un stock de pruneaux en fonction de leur position par rapport aux caisses de fruits secs et de la dose d'attractif déposée dans chaque diffuseur.

Efficiency of sexual pheromone traps in catching the Indian meal moth in a store of dried prunes in relation with the location of the traps with regard to the boxes of prunes. 
qui se déclare et renseigne sur l'opportunité de traitements de désinsectisation lorsqu'ils sont possibles. Le doublement du nombre de captures tous les jours pendant $3 \mathrm{j}$ est le critère pratique qui doit déclencher les interventions chimiques ou physiques de lutte.

\section{B. Le piégeage en masse}

En intensifiant le piégeage, le nombre total des captures augmente et, selon la théorie de NAKAMURA \& OYAMA (1978) pour les entrepôts ayant des échanges limités avec l'extérieur, il est possible de décimer les populations jusqu'à l'extinction presque totale. Bien que l'expérience pratique de cette méthode de piégeage intensif soit limitée pour l'instant à 2 années consécutives de travail dans un entrepôt servant au stockage des semences de graines potagères et horticoles, on peut remarquer aujourd'hui que la limitation des interventions insecticides dans les locaux est une réalité dès la $2^{\mathrm{e}}$ année. En effet, ces applications peuvent être mieux localisées dans le temps et dans l'espace grâce à l'information donnée par le piégeage (fig. 4). Cette mise en pratique d'un modèle théorique de contrôle du comportement d'insectes des denrées (BURKHOLDER, 1981) montre que la résolution d'une situation sanitaire délicate - car altérée régulièrement par l'entrée en entrepôt de lots de semences infestées est possible en combinant le piégeage en masse avec des traitements chimiques légers d'assurance, localisés aux zones attaquées par les ravageurs signalées par le nombre de captures dans les pièges.

Les traitements insecticides totaux, voire la fumigation, ne se justifient plus que dans des cas graves d'infestation massive ou dans certaines circonstances particulières en l'absence de moyens d'isolement, même temporaire, des lots à désinsectiser. Cette situation est hautement improbable après la mise en place du système de surveillance avec les pièges à phéromone. L'allure des courbes de captures indique que le dispositif de piégeage doit être installé avant la période des infestations, soit vers le mois d'avril dans les entrepôts non chauffés. Ceci permet d'éviter la première pointe de captures qui se manifeste dès le

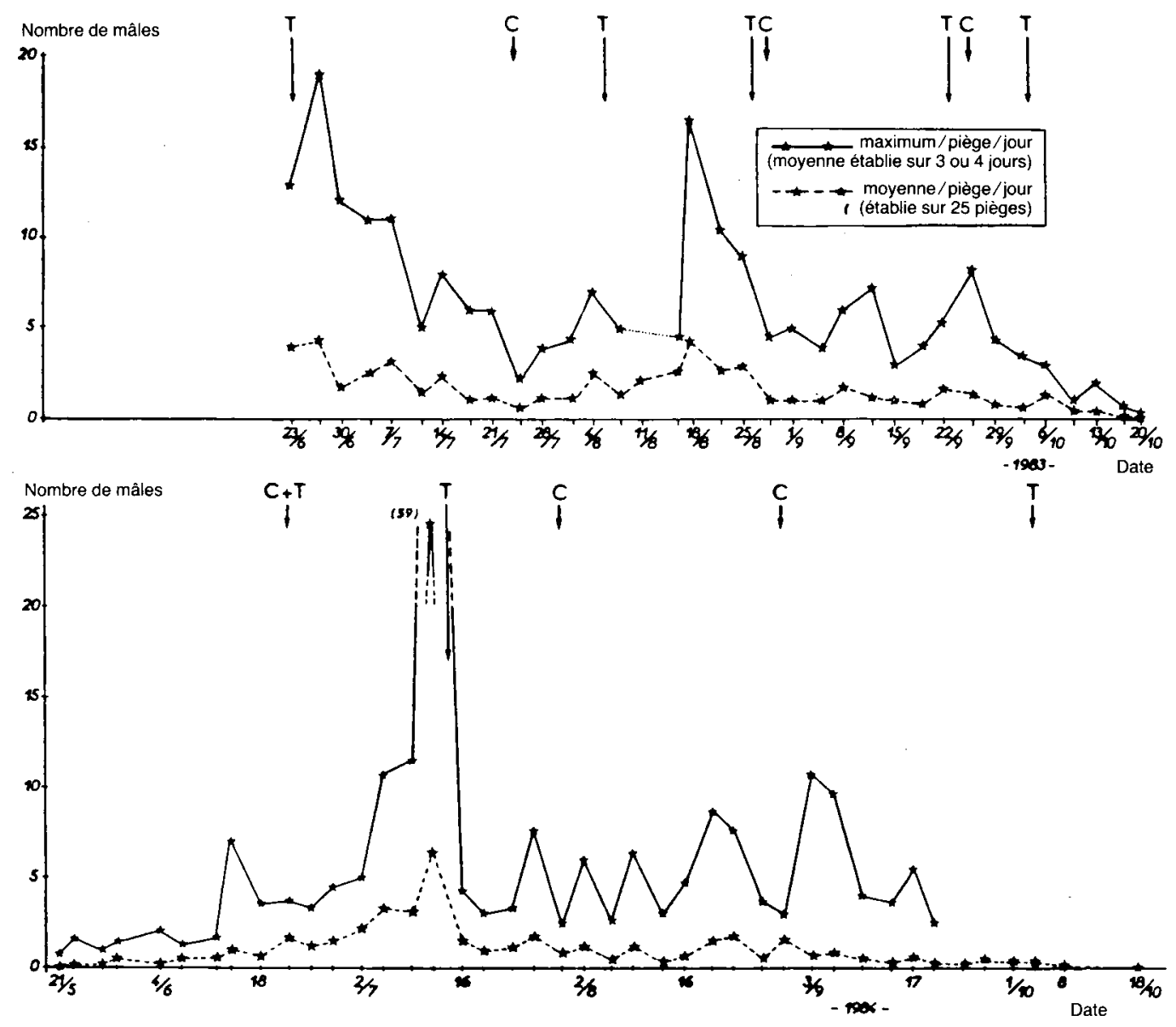

Figure 4

Courbe de piégeage en masse en 1983 (en haut) et 1984 (en bas) des mâles de P. interpunctella dans un local d'entreposage de semences potagères et horticoles régulièrement approvisionné.

- La courbe en trait plein correspond au nombre maximal de captures enregistré à chaque contrôle (ramené à une période d'un jour de piégeage).

- La courbe en pointillé correspond au nombre moyen de captures dans chaque piège par jour.

- Les approvisionnements conditionnent l'infestation et les pointes de piégeage correspondent à l'entrée en stock d'un lot de graines infestées.
- $C$ : changement des diffuseurs.

- $T$ : traitement insecticide du local par fumigène (malathion + DDVP + pyréthrines).

Number of $\mathrm{P}$. interpunctella males caught with pheromone traps during the years 1983 (above) and 1984 (below) in a warehouse regularly stocked with vegetables and flower seeds.

$C=$ dispenser renewed. $\quad T=$ insecticide treatment. 
dépôt des pièges et qui correspond à une «épuration » initiale de la population de papillons mâles déjà actifs comme cela s'est produit en 1983 (fig. 4).

Il est cependant à craindre que, dans d'autres situations d'entreposage notamment de denrées alimentaires, l'utilisation d'insecticides ou de fumigants ne soit pas aussi facile et que le système d'intensification du piégeage au moment de la détection d'un état sanitaire général en dégradation devienne le seul recours possible.

Dans ces conditions, le piégeage intensif le mieux adapté à la denrée à protéger correspondrait à la densité d'un piège pour un volume maximum de $50 \mathrm{~m}^{3}$ d'entrepôt à protéger contre 1 piège pour $350 \mathrm{~m}^{3}$ dans le cas que nous avons étudié.

\section{La confusion sexuelle}

C'est d'abord en laboratoire que l'effet de confusion sexuelle en espace limité a été vérifié (PIMAUD et al., 1976). Avec des doses journalières diffusées (calculées théoriquement) de $100 \mathrm{mg} / \mathrm{m}^{3}$, il paraissait possible d'inhiber totalement la rencontre des sexes de $P$. interpunctella. Cet effet avait été avancé en théorie alors que les mécanismes physiologiques mis en jeu ne sont pas encore bien élucidés aujourd'hui.

Un essai en grandeur réelle a été projeté pour vérifier la validité des observations faites en volume limité dans des appareils de laboratoire spécialement conçus à cet effet (CARLES et al., 1979).

Deux expériences en entrepôt de stockage de pruneaux d'Agen réalisées à une année d'intervalle ont pu être menées avec des diffuseurs passifs contenant des doses de 20 ou de $60 \mathrm{mg}$ de phéromone. On a montré que, dans les conditions de forte infestation naturelle rencontrées dans ces 2 cas, le taux de réduction des accouplements ne dépassait pas 32 p. 100 (FLEURATLESSARD et al., 1976).

Le comportement des papillons dans les jours qui suivent l'émergence n'est pas favorable à une lutte curative avec le procédé de confusion. Même si le coût de production du produit de synthèse était réduit de façon très importante, la confusion sexuelle des pyrales Phycitinae des denrées stockées ne pourrait convenir qu'en tant que mesure préventive à une éventuelle infestation.

\section{CONCLUSION}

Le piégeage sexuel des papillons mâles de Phycitinae nuisibles dans les denrées alimentaires paraît être une méthode de surveillance de l'activité de ces insectes peu onéreuse et capable de fournir de précieux renseignements d'ordre biologique pour décider de l'opportunité d'applications insecticides.

Compte tenu des conditions actuelles de production et pour des raisons de prix de revient, la confusion sexuelle ne paraît pas réalisable en pratique malgré le contexte favorable créé par la délimitation du volume à protéger et les bons résultats de perturbation de la rencontre des sexes obtenus en laboratoire avec les pyrales des denrées stockées. Par contre, le piégeage en masse, qui permet d'associer surveillance et destruction progressive de la population infestante (mâle), paraît un recours intéressant dans tous les cas où des difficultés pratiques s'opposent à l'utilisation d'insecticides pour l'assainissement des locaux, notamment lorsque les entrepôts sont occupés par des denrées consommables pendant toute l'année.

La meilleure connaissance des risques encourus obtenue avec cette nouvelle méthode d'investigation permet de programmer les périodes de désinsectisation avec la localisation du foyer à traiter. Avec une telle amélioration de la précision, le suivi de l'état sanitaire des entrepôts pour les attaques des principaux lépidoptères nuisibles devient plus aisé, les contaminations pouvant être décelées et jugulées à des seuils faibles.

Le développement de la technique au plan pratique doit obligatoirement passer par la fabrication d'un matériel de capture bien adapté au comportement de l'espèce à piéger et à la nature de la denrée entreposée avec un coût le moins élevé possible.

Cet objectif semble avoir été atteint en France (et dans quelques autres pays voisins) avec les pièges expérimentaux actuellement en service dans de nombreuses situations de stockage présentant des risques d'infestation par les Phycitinae.

Ces résultats devraient concourir à une stimulation des responsables de l'état sanitaire et de l'hygiène des entrepôts de conditionnement ou de stockage de denrées alimentaires pour l'utilisation de ce nouveau moyen de diagnostic et de lutte préventive.

\section{Recu le 21 octobre 1985.} Accepté le 3 mars 1986.

\section{REMERCIEMENTS}

La réalisation d'une partie des travaux du Laboratoire des Insectes des Denrées stockées de l'I.N.R.A. sur le sujet que nous venons de développer n'a été rendue possible qu'avec la participation financière du Bureau Interprofessionnel du Pruneau, qui nous a soutenu pendant de nombreuses années.

\section{RÉFÉRENCES BIBLIOGRAPHIQUES}

Brady U. E., 1969. Inhibition of the behavioral response of males of Indian meal moths, Plodia interpunctella and related species to female sex pheromones by exposures to sex pheromones of unrelated species. J. GA. entomol. Soc., 3, 41-45.

Brady U. E., Daley R. C., 1972. Identification of a sex pheromone from the female raisin moth Cadra figulilella. Ann. entomol. Soc. Am., 65, 6, 1356-1358.
Brady U. E., Tumlinson J. H., Brownlee R. G., Silverstein R. M., 1971. Sex stimulant and attractant in the Indian meal moth and in the almond moth. Science, 171, 802-804.

Burkholder W. E., 1981. Biomonitoring for stored-product insects, 4, 29-40. In Mitchell E. R. : «Management of insect pests with semio-chemicals : concepts and practice». Plenum Press New York, 514 p. 
Carles J. P., Fleurat-Lessard F., Roehrich R., 1979. Un appareil pour les essais biologiques de comportement de lépidoptères exposés à des doses élevées d'attractif sexuel. Biol. Behav., 4, 3, 205-217.

Fleurat-Lessard F., 1983. Les phéromones des insectes des denrées : possibilités d'utilisation pratique dans le domaine de l'après-récolte. Bull. C.I.L.D.A., 14, $29-41$.

Fleurat-Lessard F., Pimaud M. F., Cangardel H., 1976. Effets de doses élevées de ZETA sur Plodia interpunctella (Hübner) (Lep. Pyralidae) dans les stocks de pruneaux d'Agen. C. R. $I^{\text {res }}$ journées phéromones, I.N.R.A., Bordeaux, 163-169.

Haines C. P., 1976. The use of synthetic sex pheromones for pest management in stored product situations. Pestic. Sci., 7, 647-649.

Kuwahara Y., Kitamura C., Takahashi S., Hara H., Fukami H., 1971 $a$. Sex pheromone of the almond moth and the Indian meal moth : cis-9, trans-12-tetradecadienyl acetate. Science, 171, 801802.

Kuwahara Y., Hara H., Ishii S., Fukami H., 1971b. The sex pheromone of the Mediterranean flour moth. Agr. Biol. Chem., 35, 3, 447-448.

Le Torc'h J. M., Fleurat-Lessard F., 1980. Piégeage sexuel de la teigne des fruits secs Plodia interpunctella $\mathrm{Hb}$. en entrepôt de stockage traditionnel de pruneaux. C. R. $3^{e}$ journées phéromones, I.N.R.A., Colmar, 105-106.

Levinson H. Z., Levinson A. R., 1979. Trapping of storage insects by sex and food attractants as a tool of integrated control, 327-341. In J. F. Ritter : "Chemical ecology : odours communication in animals ». Elsevier, Amsterdam, $427 \mathrm{p}$.
Mankin R. W., 1979. Attraction of insects to odorant sources in a warehouse. Thèse Ph. D. Univ. of Florida, 79 p. (In : Diss. Abstr., B, Sci. Eng., 1980, 40, 9).

Nakamura K., Oyama M., 1978. An equation for the competition between pheromone traps and adult females. Appl. entomol. Zool., 13, 2, 176-184.

Pimaud M. F., Cangardel H., Fleurat-Lessard F., 1976. Effets de fortes doses de ZETA en milieu confiné sur la reproduction de Plodia interpunctella Hübner (Lépidoptère Pyralidae) en laboratoire. C. R. $I^{\text {res }}$ journées phéromones, I.N.R.A., Bordeaux, 129-135.

Read J. S., Beevor P. S., 1976. Analytical studies on the sex pheromone complex of Ephestia cautella (Walker) (Lepidoptera : Phycitinae). J. stored Prod. Res., 12, 55-57.

Vick K. W., Sower L. L., 1973. Z-9,Z-12-tetradecadien-1-ol acetate : an inhibitor, of the response to the sex pheromone of Plodia interpunctella. J. econ. Entomol., 66, 1258-1260.

Vick K. W., Coffelt J. A., Mankin R. W., Soderstrom E. L., 1981. Recent developments in the use of pheromones to monitor Plodia interpunctella and Ephestia cautella, 19-28. In E. R. Mitchell : "Management of insect pests with semiochemicals ». Plenum Press, New York, 514 p. 